\title{
Variáveis dendrométricas e produção de Eucalyptus benthamii em diferentes espaçamentos
}

\author{
Luciano Farinha Watzlawick ${ }^{1}$, Cristiane Carla Benin ${ }^{2}$ \\ ${ }^{1}$ Universidade Estadual do Centro- Oeste, Guarapuava-PR. ${ }^{2}$ Universidade Estadual do Centro-Oeste, Guarapuava-PR. \\ E-mail: farinha@unicentro.br
}

\begin{abstract}
Resumo
Neste estudo objetivou-se avaliar o efeito do espaçamento de plantio na produção e nas variáveis dendrométricas, em plantio experimental com sementes melhoradas da espécie $E$. benthamii, aos seis anos de idade. $O$ delineamento experimental adotado foi em blocos ao acaso, com quatro repetições de vinte árvores. Os tratamentos foram quatro espaçamentos de plantio $3 \times 2 \mathrm{~m}, 3 \times 3 \mathrm{~m}, 3 \times 4 \mathrm{~m}$ e $4 \times 4 \mathrm{~m}$. Após os procedimentos em campo (inventário florestal, amostragem e cubagem de 79 árvores), obtiveram-se os principais parâmetros dendrométricos em cada espaçamento. As alturas foram determinadas por relação hipsométrica e o volume das demais árvores do povoamento foi estimado pelo modelo de Schumacher $\mathrm{e}$ Hall (1933). As variáveis dendrométricas apresentaram alta correlação com o espaçamento. Os espaçamentos mais amplos favoreceram o diâmetro, altura, área transversal e o volume individual, enquanto os menores espaços vitais foram responsáveis pelos maiores valores de área basal e volume por hectare. A produção oscilou de $238,90 \mathrm{~m}^{3} \mathrm{ha}^{-1}$ no maior espaçamento a $392,08 \mathrm{~m}^{3} \mathrm{ha}^{-1}$ no menor espaçamento. Concluiu-se que houve efeito do espaçamento do plantio, e que com as tendências observadas quanto a maior produção por hectare nos espaçamentos mais adensados é oportuno planejar a produção florestal em função do espaçamento de plantio adotado.
\end{abstract}

Palavra- chave: espaço vital; florestas plantadas; manejo florestal.

\section{Dendrometric variables and Eucalyptus benthamii production in different spaces}

\begin{abstract}
This study aimed to evaluate the effect of planting spacing on production and dendrometric variables, in experimental planting with improved $E$. benthamii seeds, at six years of age. The experimental design adopted was in randomized blocks, with four replications of twenty trees. The treatments were four planting spacing $3 \times 2 \mathrm{~m}, 3 \times 3 \mathrm{~m}, 3 \times 4 \mathrm{~m}$ and $4 \times 4 \mathrm{~m}$. After the field procedures (forest inventory, sampling and cubage of 79 trees), the main dendrometric parameters were obtained in each spacing. The heights were determined by a hypsometric relationship and the volume of other trees in the stand was estimated by the model of Schumacher and Hall (1933). The dendrometric variables showed a high correlation with the spacing. The wider spacing favored the diameter, height, transversal area and the individual volume, while the smaller vital spaces were responsible for the highest values of basal area and volume per hectare. Production ranged from $238.90 \mathrm{~m}^{3} \mathrm{ha}^{-1}$ in the largest spacing to $392.08 \mathrm{~m}^{3} \mathrm{ha}^{-1}$ in the smallest spacing. It was concluded that there was an effect of the planting spacing, and that with the trends observed regarding the higher production per hectare in the denser spacing, it is opportune to plan the forest production according to the planting spacing adopted.
\end{abstract}

Keyword: vital space; planted forests; forest management. 


\section{Introdução}

As florestas plantadas são uma importante atividade econômica, social e ambiental. $O$ retorno da cadeia produtiva de base florestal e a busca pela sustentabilidade na utilização dos recursos naturais e nos sistemas de produção enfatizam a utilização de matéria-prima florestal proveniente de plantios florestais. Associado a este cenário, a capacidade de produção, utilização da madeira e o ciclo de corte, são fatores essenciais na definição da espécie a ser plantada. Assim, devido as amplas possibilidades de utilização de sua madeira, rápido crescimento e excelente produtividade, as espécies do gênero Eucalyptus spp. têm relevância na composição dos reflorestamentos em escala comercial, representando 6,97 milhões de hectares no Brasil (IBÁ, 2020).

O Eucalyptus benthamii é uma opção de espécie para reflorestamentos em regiões de clima frio, onde ocorre geadas frequentes e severas, como no sul do Brasil (GRAÇA et al., 1999). Assim, na região sul do Brasil, a espécie Eucalyptus benthamii Maiden et Cambage vêm sendo plantada em maior escala nos últimos anos. As características silviculturais e de modo singular a tolerância à incidência de frequentes geadas no inverno, destacam o uso da espécie nesta região.

Avaliações realizadas em árvores de primeira geração de $E$. benthamii, no município de Colombo-PR, indicaram bom desempenho da espécie, com incremento médio anual de 2,3 metros em altura e $2,7 \mathrm{~cm}$ em diâmetro (PEREIRA et al., 2000). No estado de Santa Catarina, plantios experimentais de $E$. benthamii com dois e três anos de idade mostraram bom crescimento e resistência à ocorrência de geadas severas e frequentes (HIGA; PEREIRA, 2003).

Além das particularidades da espécie e da região em que se implanta uma floresta, outro fator decisivo da produção florestal concerne ao espaçamento de plantio, haja vista que esta é a primeira prática que pode causar alterações nas características da futura árvore (KRONKA et al., 2005). O espaçamento determina a forma de crescimento de uma floresta e algumas tendências no comportamento dos parâmetros dendrométricos (altura, diâmetro, área basal, volume individual e volume/ha) (LEITE et al., 2006). A definição do espaçamento deve ser feita de acordo com as especificidades do local (características do solo e fatores climáticos), espécie (forma de crescimento e desenvolvimento radicial, a tolerância e a adaptabilidade da espécie escolhida), práticas silviculturais e o objetivo da produção (LIMA et al., 2013).

A escolha de arranjos e espaçamentos inadequados a espécie e à finalidade do plantio podem intensificar a competição e reduzir a homogeneidade de crescimento da floresta (BINKLEY, 2002).

Desde o início dos reflorestamentos com Eucalyptus em escala comercial no Brasil, vêm-se estudando o efeito do espaçamento no crescimento das variáveis dendrométricas e suas implicações na produção florestal. $O$ comportamento de diferentes espécies face ao espaçamento adotado e novas técnicas de espaçamento (SANTOS, 2011), a interação espaçamento $x$ custos de produção e colheita (LEITE et al., 2014) e a influência do espaçamento e de outros fatores sob a qualidade do produto final e propriedades da madeira também foram investigados (ALZATE et al., 2005; SEREGHETTI, 2015) no âmbito de se encontrar respostas quanto aos efeitos do espaçamento e a determinação dos espaçamentos ideais em cada situação. Estes estudos descreveram algumas tendências do crescimento da floresta em função do espaçamento, podendo-se destacar o desenvolvimento do diâmetro, altura e volume, determinados pelo número de árvores em um hectare (ENCINAS, 2005; REINER et al., 2011).

No entanto, diante do contexto florestal atual, contemplando principalmente os aspectos do melhoramento florestal e tecnologias agregadas à formação de floresta, torna-se evidente a importância de novos ensaios com espaçamentos para conhecer a possibilidade de ganhos, seja para uma determinada espécie, para determinada região ou para a interação de condições que determinam a produção florestal.

Assim, objetivou-se avaliar o efeito de diferentes espaçamentos de plantio na produção e no desenvolvimento das variáveis dendrométricas em plantio experimental de $E$. benthamii, aos seis anos de idade, na região de Guarapuava-PR.

\section{Material e métodos}

A área de estudo está localizada no Município de Guarapuava, situada no terceiro planalto paranaense, na região Centro - Sul. O clima da região é classificado como subtropical, 
com temperaturas médias anuais entre 16 e $20^{\circ} \mathrm{C}$, com inverno frio e ocorrência de geadas severas e frequentes, sem estação seca. As chuvas são abundantes e bem distribuídas durante todo o ano. Os solos compreendidos na região de Guarapuava- PR são principalmente os latossolos, cambissolos, afloramento rochosos e neossolos litólicos (EMBRAPA, 2018).

Foram utilizadas mudas de sementes melhoradas da espécie $E$. benthamii, da cultiva EBC 06 Candói, para a implantação de um experimento, utilizando os espaçamentos de plantio $3 \times 2,3 \times 3,4 \times 3$ e $4 \times 4 \mathrm{~m}$. O ensaio foi instalado no ano de 2006, na área experimental do curso de Agronomia da Universidade Estadual do Centro- Oeste, Campus CEDETEG.

O delineamento experimental utilizado foi o de blocos ao acaso, com quatro tratamentos e quatro repetições de vinte árvores mensuráveis em cada unidade amostral. As unidades amostrais foram sorteadas e alocadas aleatoriamente dentro de cada bloco, sendo estas isoladas por linhas duplas de bordaduras (Figura 1).

Figura 1. Layout do experimento com a disposição dos blocos e alocação dos tratamentos

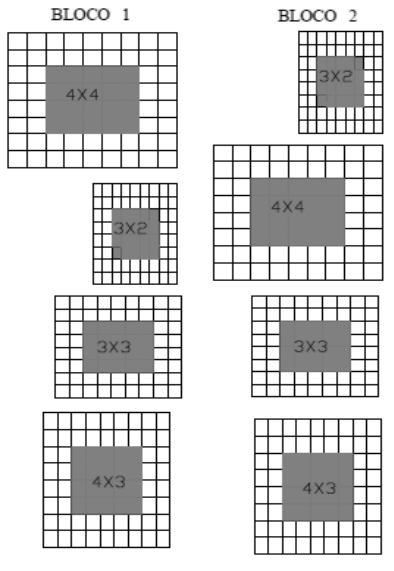

Utilizou-se o sistema de cultivo mínimo do solo, sendo as covas abertas diretamente nas linhas de plantio e plantio manual das mudas. A adubação foi realizada com NPK 04-14-08, aplicado em covetas laterais, na proporção de $200 \mathrm{~g} \mathrm{planta}^{-1}$. Os tratos culturais foram o combate a formiga após a finalização de plantio, monitoramento de formigas pós-plantio mensalmente até seis meses e a capina mecânica (coroamento das mudas) conforme necessidade, até o 1 - ano de plantio.

O presente estudo está baseado em dados dendrométricos correspondentes aos seis anos de idade do plantio, obtidos pela realização de censo. A partir disso, os diâmetros foram agrupados em classes diamétricas, utilizando-se a

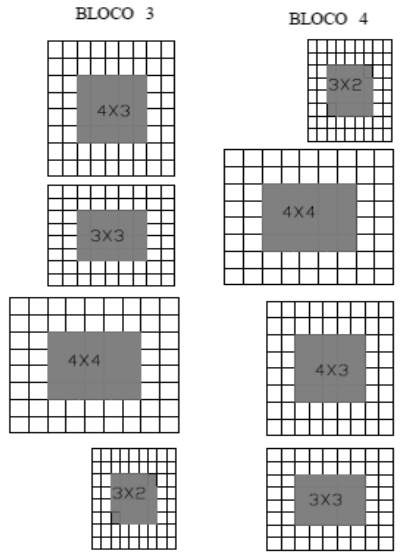

fórmula de Sturges na determinação do número de classes:

Em que:

$$
K=1+3,33 * \log (n)
$$

$\mathrm{K}=$ número de classes

$\mathrm{n}$ = número de observações

As árvores para cubagem foram selecionadas nas classes de maior frequência em cada espaçamento, amostrando-se também árvores finas e grossas nos espaçamentos que as contemplavam, totalizando 79 árvores (Tabela 1). As seções de cubagem foram definidas pelo método relativo, considerando duas posições absolutas (base e DAP). Assim, os pontos de amostragem para a tomada dos diâmetros foram: $0,10 \mathrm{~m} ; 1,30 \mathrm{~m} ; 15,25,35,45,55,65,75,85$ e $95 \%$ da altura total da árvore. O volume de cada seção foi calculado pelo método de Smalian. 
Tabela 1. Classes de diâmetro e número de árvores cubadas em cada espaçamento

\begin{tabular}{ccccc}
\hline $\begin{array}{c}\text { Centro da centro } \\
\text { diamétrica }(\mathrm{cm})\end{array}$ & $3 \times 2$ & $3 \times 3$ & $3 \times 4$ & $4 \times 4$ \\
\cline { 2 - 5 } & $\begin{array}{c}\text { Total de } \\
\text { árvores }\end{array}$ & $\begin{array}{c}\text { Total de } \\
\text { árvores }\end{array}$ & $\begin{array}{c}\text { Total de } \\
\text { árvores }\end{array}$ & $\begin{array}{c}\text { Total de } \\
\text { árvores }\end{array}$ \\
\hline$<13,47$ & 4 & 2 & 1 & 1 \\
\hline 13,47 & 3 & 3 & 4 & 4 \\
\hline 15,91 & 3 & 3 & 3 & 3 \\
\hline 18,35 & 3 & 4 & 3 & 3 \\
\hline 20,79 & 4 & 4 & 4 & 5 \\
\hline 23,23 & 3 & 3 & 5 & 0 \\
\hline$>23,23$ & 0 & 1 & 1 & 3 \\
\hline
\end{tabular}

As alturas das árvores do povoamento foram determinadas por relação hipsométrica, através da equação ajustada com as alturas reais obtidas da cubagem:

$D A P^{2}$

$$
h=1,9010+2,3217 * D A P-0,0479 *
$$

Em que:

$\mathrm{h}=$ altura $(\mathrm{m})$

$\mathrm{DAP}=$ diâmetro $\mathrm{a}$ altura do peito $(\mathrm{cm})$

O volume individual das demais árvores do povoamento foi estimado pela equação volumétrica de Schumacher e Hall ajustada, conforme descrita abaixo:

$$
\begin{aligned}
& \operatorname{Ln} V=-10,3242+1,7377 \\
& * \ln D A P+1,2060 * \ln H t
\end{aligned}
$$

Em que:

$\left(m^{3}\right)$

Ln $V$ = logaritmo neperiano do volume

Ln $\mathrm{Ht}=$ logaritmo da altura total $(\mathrm{m})$

In $\mathrm{DAP}=$ logaritmo neperiano do diâmetro à altura do peito $(\mathrm{cm})$

As estimativas de área basal e volume por hectare foram realizados multiplicando-se os valores individuais médios pelo número de indivíduos presentes em cada espaçamento avaliado.

A correlação linear entre as principais variáveis dendrométricas e espaçamento de plantio foram avaliadas pelo coeficiente de
Pearson (r). Para avaliar o efeito do espaçamento sobre os parâmetros dendrométricos, inicialmente os dados foram submetidos ao teste de normalidade e homogeneidade das variâncias (Bartlett) e em seguida procedeu-se a análise de variância (ANOVA). Quando a hipótese de nulidade foi rejeitada, as médias foram comparadas pelo Teste de Tukey. Todas as análises foram realizadas ao nível de $5 \%$ de probabilidade de erro.

\section{Resultados e discussão}

Observou-se alto coeficiente de correlação de Pearson $(0,98)$ para as variáveis: diâmetro à altura do peito, área transversal e volume individual com o espaçamento de plantio (Tabela 2). Para o volume ha ${ }^{-1}$, o coeficiente de Pearson $(-0,96)$ apontou forte correlação linear negativa (Tabela 2), o que explica a afinidade do espaçamento com a produção volumétrica $e$ também, a tendência de que a produção volumétrica $\left(\mathrm{m}^{3} \mathrm{ha}^{-1}\right)$ reduz com o aumento do espaçamento entre plantas. As correlações entre a altura total e área basal com o espaçamento não apresentaram significância estatística ( $p$ > $0,05)$. Em relação à área basal isso pode estar associado à idade do plantio, pois ao se tratar de espaçamentos mais amplos, as plantas ainda estão em processo de ocupação da área e não há indícios de competição entre elas. 
Tabela 2. Correlação linear entre as variáveis dendrométricas e o espaçamento de plantio, em plantio experimental de E. benthamii, aos seis anos de idade.

\begin{tabular}{lcc}
\hline \multicolumn{1}{c}{ Variáveis } & $\mathrm{r}$ & $\mathrm{p}$ - valor \\
\hline Altura total $(\mathrm{m}) \times$ espaçamento de plantio $\left(\mathrm{m}^{2}\right)$ & 0,92 & $0,0793^{\text {ns }}$ \\
DAP $(\mathrm{cm}) \times$ espaçamento de plantio $\left(\mathrm{m}^{2}\right)$ & 0,98 & $0,0232^{*}$ \\
Área transversal $\left(\mathrm{m}^{2}\right)$ x espaçamento de plantio $\left(\mathrm{m}^{2}\right)$ & 0,98 & $0,0181^{*}$ \\
Área basal $\left(\mathrm{m}^{2} \mathrm{ha}^{-1}\right) \times$ espaçamento de plantio $\left(\mathrm{m}^{2}\right)$ & $-0,93$ & $0,0676^{\text {ns }}$ \\
Volume individual $\left(\mathrm{m}^{3}\right.$ arv $\left.{ }^{-1}\right)$ x espaçamento de plantio $\left(\mathrm{m}^{2}\right)$ & 0,98 & $0,0160^{*}$ \\
Volume total ha- ${ }^{1}\left(\mathrm{~m}^{3} \mathrm{ha}^{-1}\right) \times$ espaçamento de plantio $\left(\mathrm{m}^{2}\right)$ & $-0,96$ & $0,0357^{*}$ \\
\hline
\end{tabular}

$r=$ Coeficiente de correlação de Pearson. ns- não significativo ao nível de $5 \%$ de probabilidade de erro.

Os resultados da Figura 2 representam a distribuição diamétrica em plantio de $E$. benthamii, para os diferentes espaçamentos. Nos maiores espaçamentos há maior número de indivíduos pertencentes às maiores classes de diâmetro, sendo que foi possível observar gradativo aumento no número de indivíduos na classe de $30 \mathrm{~cm}$, dos espaçamentos $3 \times 3 \mathrm{~m}$ e $4 \mathrm{x}$ $3 \mathrm{~m}$ para $\mathrm{o}$ espaçamento $4 \times 4 \mathrm{~m}$.

Figura 2. Distribuição diamétrica em plantio experimental de E. benthamii, aos seis anos de idade, em função de diferentes espaçamentos de plantio.
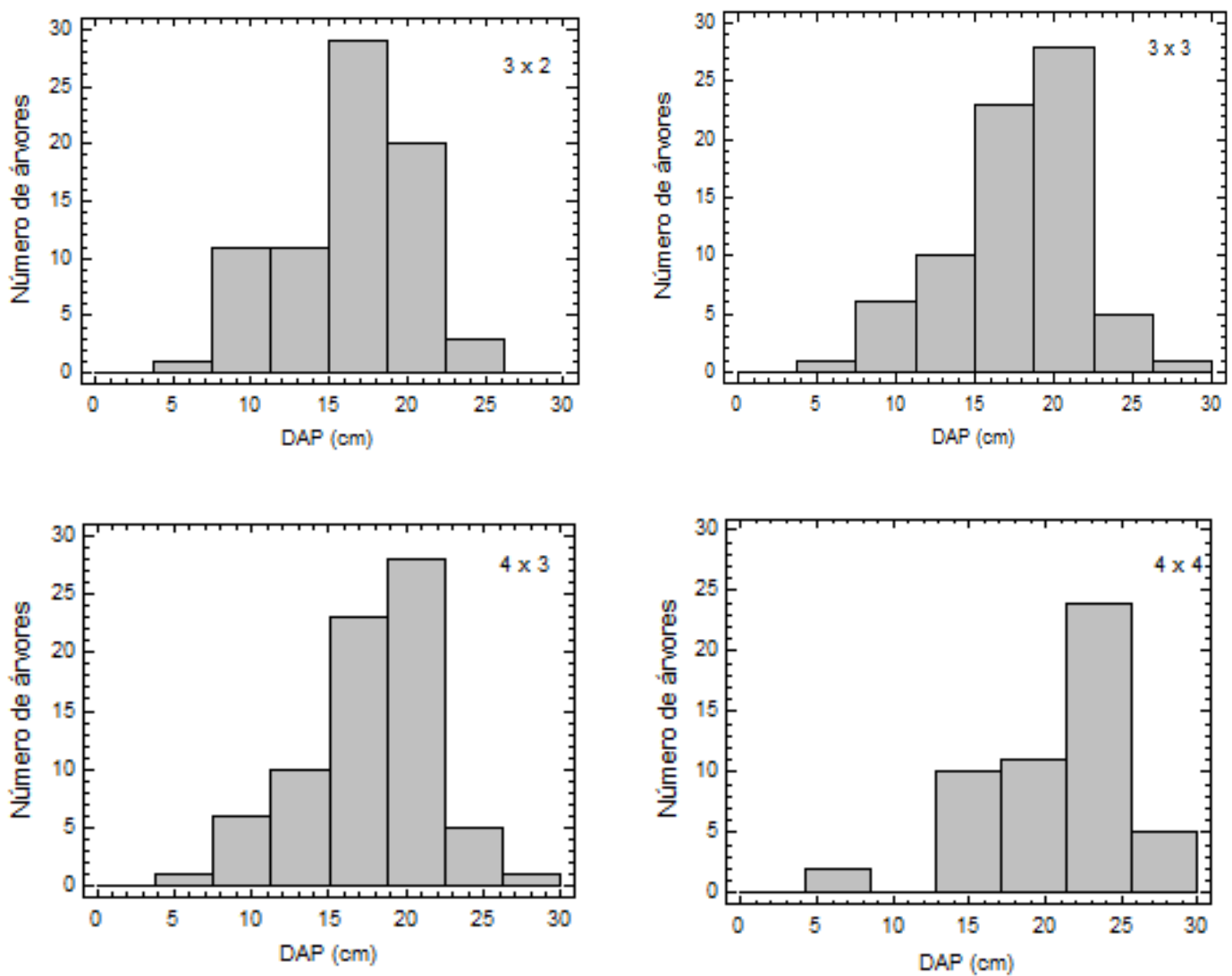

No espaçamento $3 \times 2 \mathrm{~m}$, houve poucos indivíduos presentes na classe de $25 \mathrm{~cm}$, ao se comparar com o espaçamento $4 \times 4 \mathrm{~m}$. Isso mostra a influência positiva da disponibilidade de espaço ao desenvolvimento do diâmetro nos espaçamentos mais amplos. No espaçamento $4 \mathrm{x}$ $4 \mathrm{~m}$, não houve indivíduos na classe de $10 \mathrm{~cm}$ de

diâmetro e poucos indivíduos na classe de $5 \mathrm{~cm}$, indicando baixa ocorrência de indivíduos com diâmetro inferior a $10 \mathrm{~cm}$, na idade avaliada.

Para a altura total, no menor espaçamento avaliado $(3 \times 2 \mathrm{~m})$, registrou-se o menor valor médio $(22,35 \mathrm{~m})$. Já no espaçamento $3 \times 3 \mathrm{~m}$, a altura média foi de $23,36 \mathrm{~m}$, o qual não 
diferiu estatisticamente dos espaçamentos $3 \times 2$ $\mathrm{m}, 4 \times 3 \mathrm{~m}$ e $4 \times 4 \mathrm{~m}$. Não houve diferença estatística entre os espaçamentos $4 \times 3 \mathrm{~m}$ e $4 \times 4$ $\mathrm{m}$ para o crescimento em altura. Ao contrário da tendência citada na literatura, de que os espaçamentos maiores entre plantas apresentam tendência de redução da altura média (INOUE et al., 2011), neste estudo, os maiores espaçamentos foram os que apresentaram as maiores alturas, nas avaliações aos seis anos de idade.

Silva (2005) e Santos (2011) também relataram sobre maiores alturas nos espaçamentos com maior área útil entre plantas, comprovando que em que alguns casos a altura média das árvores tende a aumentar de acordo com a amplitude do espaçamento.

Aos seis anos de idade, observou-se efeito positivo dos espaçamentos mais amplos no desenvolvimento do diâmetro das árvores de $E$. benthamii. Os maiores valores foram de $20,64 \mathrm{~cm}$ no espaçamento $4 \times 4 \mathrm{~m}$ e $19,80 \mathrm{~cm}$ no espaçamento $4 \times 3 \mathrm{~m}$ (Tabela 3 ). Os valores médios de área transversal por árvore apresentaram diferenças estatísticas entre o espaçamento mais amplo e o espaçamento reduzido, confirmando o efeito do espaçamento sobre o desenvolvimento desta variável (Tabela 3).

Tabela 3. Altura $(\mathrm{m}), \mathrm{DAP}(\mathrm{cm})$ e área transversal $\left(\mathrm{m}^{2}\right)$ de árvores de $E$. benthamii em plantio experimental, aos seis anos de idade, em função de quatro espaçamentos.

\begin{tabular}{ccccc}
\hline Espaçamento $(\mathrm{m})$ & $\begin{array}{c}\text { Altura total } \\
(\mathrm{m})\end{array}$ & $\begin{array}{c}\text { DAP } \\
(\mathrm{cm})\end{array}$ & $\begin{array}{c}\text { Área transversal } \\
\left(\mathrm{m}^{2}\right)\end{array}$ \\
\hline $3 \times 2$ & $22,35 \mathrm{~b}$ & $16,19 \mathrm{c}$ & 0,0230 & $\mathrm{c}$ \\
$3 \times 3$ & $23,36 \mathrm{ab}$ & $17,64 \mathrm{bc}$ & 0,0257 & $\mathrm{bc}$ \\
$4 \times 3$ & $24,58 \mathrm{a}$ & $19,80 \mathrm{ab}$ & 0,0319 & $\mathrm{ab}$ \\
$4 \times 4$ & $24,62 \mathrm{a}$ & $20,64 \mathrm{a}$ & $0,0349 \mathrm{a}$ \\
\hline & 3,03 & & & \\
\hline
\end{tabular}

Médias seguidas de mesma letra minúscula não diferem estatisticamente pelo teste de Tukey ao nível de $5 \%$ de probabilidade de erro. CV \% - Coeficiente de variação.

O espaçamento mais adensado neste estudo $(3 \times 2 \mathrm{~m})$ apresentou valor médio do diâmetro inferior aos demais espaçamentos $(16,19 \mathrm{~cm})$, no entanto não diferiu estatisticamente do valor médio encontrado no espaçamento $3 \times 3 \mathrm{~m}$, cujo valor foi de 17,64 cm.

Este resultado corrobora com os estudos de Leles et al. (2001), Berger et al. (2002), Sanqueta et al. (2003), Muller et al. (2005) e Inoue et al. (2011), dentre outros que observaram que os espaçamentos mais amplos potencializaram o desenvolvimento do diâmetro das árvores. Nestas situações, o maior crescimento em diâmetro está associado ao eficiente aproveitamento dos recursos disponibilizados a cada planta, sem que haja competição por espaço, luz, água e nutrientes.

Nos espaçamentos mais amplos $4 \times 4 \mathrm{~m} \mathrm{e}$ $4 \times 3 \mathrm{~m}$, as áreas transversais médias foram de 0,0349 e 0,0319 $\mathrm{m}^{2}$, enquanto no menor espaçamento estudado o valor médio registrado foi de $0,0230 \mathrm{~m}^{2}$. Isso mostra a diferença na taxa de ocupação de cada árvore em função do diâmetro da árvore, o qual é determinado pelo espaçamento de plantio adotado.

As áreas transversais médias dos espaçamentos $3 \times 3 \mathrm{~m}\left(0,0257 \mathrm{~m}^{2}\right)$ e $4 \times 3 \mathrm{~m}$ $\left(0,0319 \mathrm{~m}^{2}\right)$ não diferiram estatisticamente. Ainda, foram estatisticamente iguais as áreas transversais dos espaçamentos $3 \times 2 \mathrm{~m}$ e $3 \times 3 \mathrm{~m}$ e dos espaçamentos $4 \times 3 \mathrm{~m}$ e $4 \times 4 \mathrm{~m}$.

Nas estimativas da área basal no plantio experimental de E. benthamii, aos seis anos de idade, notou-se um decréscimo dos valores médios de área basal $\left(\mathrm{m}^{2} \mathrm{ha}^{-1}\right)$ em função do aumento do espaçamento de plantio. Os valores variaram de 38,$35 ; 28,56 ; 26,6$ e $21,51 \mathrm{~m}^{2} \mathrm{ha}^{-1}$, para os respectivos espaçamentos $3 \times 2 \mathrm{~m} ; 3 \times 3$ $\mathrm{m} ; 4 \times 3 \mathrm{~m}$ e $4 \times 4 \mathrm{~m}$. Os valores médios de área basal para os espaçamentos $4 \times 4 \mathrm{~m}$ e $4 \times 3 \mathrm{~m}$ foram estatisticamente iguais. $O$ espaçamento $4 x$ $3 \mathrm{~m}$ não diferiu estatisticamente do espaçamento $3 \times 3 \mathrm{~m}$ em termos de área basal (Tabela 4). 
Tabela 4. Valores médios de área basal $\left(\mathrm{m}^{2}\right.$ ha $\left.{ }^{-1}\right)$, volume individual $\left(\mathrm{m}^{3} \operatorname{arv}^{-1}\right)$ e volume por hectare $\left(\mathrm{m}^{3}\right.$ ha

${ }^{1}$ ) em plantio experimental de $E$. benthamii, aos seis anos de idade, em função de quatro espaçamentos.

\begin{tabular}{cccc}
\hline Espaçamento $(\mathrm{m})$ & $\begin{array}{c}\text { Área basal } \\
\left(\mathrm{m}^{2} \mathrm{ha}^{-1}\right)\end{array}$ & $\begin{array}{c}\text { Volume individual } \\
\left(\mathrm{m}^{3} \mathrm{arv}^{-1}\right)\end{array}$ & $\begin{array}{c}\text { Volume. ha } \\
\left(\mathrm{m}^{3} \mathrm{arv}^{-1}\right)\end{array}$ \\
\hline $3 \times 2$ & $38,35 \mathrm{a}$ & $0,2353 \mathrm{c}$ & $392,08 \mathrm{a}$ \\
$3 \times 3$ & $28,56 \mathrm{~b}$ & $0,2805 \mathrm{bc}$ & $311,68 \mathrm{ab}$ \\
$4 \times 3$ & $26,60 \mathrm{bc}$ & $0,352 \mathrm{ab}$ & $293,50 \mathrm{~b}$ \\
$4 \times 4$ & $21,51 \mathrm{c}$ & $0,3856 \mathrm{a}$ & $238,90 \mathrm{~b}$ \\
\hline CV (\%) & 9,58 & 11,51 & 13,09 \\
\hline
\end{tabular}

Médias seguidas de mesma letra minúscula não diferem estatisticamente pelo teste de Tukey ao nível de $5 \%$ de probabilidade de erro. CV \%- Coeficiente de variação.

A maior estimativa de área basal encontrada no espaçamento mais reduzido foi favorecida pela presença de maior número de indivíduos por unidade de área. Segundo Schneider (1993), a área basal em plantios florestais é dependente do diâmetro médio e do número de indivíduos por unidade de área. Morais (2006) constatou valores médios de área basal decrescentes com $O$ aumento do espaçamento, em plantio de $E$. camaldulensis $\times E$. urophylla, na região noroeste de Minas Gerais. Nesta ocasião, o valor mais pronunciado para a variável foi verificado no espaçamento com área útil de $6 \mathrm{~m}^{2}\left(31,11 \mathrm{~m}^{2} \mathrm{ha}^{-1}\right)$.

Em Pinus taeda, até os oito anos de idade, as maiores estimativas de área basal foram verificadas conforme o menor espaçamento entre plantas (LEITE et al., 2006). A mesma tendência no comportamento da área basal foi registrada para $E$. nitens, onde os maiores valores médios de área basal foram registrados para os espaçamentos mais adensados (PINKARD; NEILSEN, 2003).

Em relação ao volume médio individual, houve diferença estatística entre o espaçamento mais amplo e o espaçamento mais reduzido (Tabela 4). As árvores do espaçamento com $4 \times 4$ $\mathrm{m}$ apresentaram o maior volume individual $\left(0,3856 \mathrm{~m}^{3} \mathrm{arv}^{-1}\right)$, seguido do espaçamento $3 \times 4$ $\mathrm{m}\left(0,3523 \mathrm{~m}^{3}\right.$ arv $\left.^{-1}\right)$, no entanto, não registrou-se diferença estatística entre estes espaçamentos. 0 volume individual verificado nos espaçamentos 4 x $3 \mathrm{~m}$ e $3 \times 3 \mathrm{~m}$ também foram estatisticamente semelhantes. Para o espaçamento mais reduzido registrou-se $0,2353 \mathrm{~m}^{3} \mathrm{arv}^{-1}$, não diferindo do volume encontrado no espaçamento com $3 \times 3$ $\mathrm{m}$, cujo valor médio foi de $0,2805 \mathrm{~m}^{3} \mathrm{arv}^{-1}$.

O resultado desta variável vem ao encontro do trabalho de Berger et al. (2002), no qual os autores verificaram que os maiores volumes comerciais individuais para um clone de E. saligna corresponderam aos espaçamentos mais amplos, independentemente da idade de avaliação. Silva (2005) observou que o volume individual de indivíduos híbridos $E$. grandis $x E$. urophylla no maior espaçamento $\left(15 \mathrm{~m}^{2}\right.$ planta $\left.^{-1}\right)$ foi praticamente o dobro do menor espaçamento adotado em seu estudo ( $6 \mathrm{~m}^{2}$ planta $\left.{ }^{-1}\right)$ nas avaliações do clone aos seis anos de idade, no Município de Altinópolis- SP. Magalhães et al. (2006) também confirmaram que o volume individual foi mais expressivo com o aumento do espaço vital entre plantas para as quatro espécies de Eucalyptus estudadas.

$\mathrm{O}$ volume médio por hectare no menor espaçamento $(3 \times 2 \mathrm{~m})$ foi de $392,08 \mathrm{~m}^{3} \mathrm{ha}^{-1}$ enquanto o espaçamento $3 \times 3 \mathrm{~m}$ apresentou volume médio de $311,68 \mathrm{~m}^{3}$ ha. Estes resultados mostram que para esta variável $\left(\right.$ vol ha $\left.{ }^{-1}\right)$, os espaçamentos $3 \times 2 \mathrm{~m}$ e $3 \times 3 \mathrm{~m}$ são estatisticamente iguais (Tabela 4). A produção volumétrica no espaçamento $3 \times 2 \mathrm{~m}$ foi cerca de $39 \%$ superior em relação ao espaçamento $4 \times 4$ $\mathrm{m}$ e $25,14 \%$ superior à produção média do espaçamento com $4 \times 3 \mathrm{~m}$.

O espaçamento $4 \times 4 \mathrm{~m}$, embora apresentando árvores com maior volume médio individual, teve a menor produção volumétrica registrada $\left(238,90 \mathrm{~m}^{3} \mathrm{ha}^{-1}\right)$, no entanto não diferiu do espaçamento com $4 \times 3 \mathrm{~m}$, no qual a produção foi igual a $293,5 \mathrm{~m}^{3} \mathrm{ha}^{-1}$, na idade avaliada.

Lima et al. (2013) verificou menor produtividade volumétrica no espaçamento com $16 \mathrm{~m}^{2}$ de área vital em um plantio experimental de Pinus taeda L., implantados em nove diferentes espaçamentos. O autor associa esta observação ao menor número de árvores por hectare, com a adoção deste espaçamento. Para E. grandis a menor densidade populacional 
causou efeitos negativos na produção volumétrica. No entanto, estes efeitos sobre a produtividade foram minimizados com $O$ progresso da idade da floresta (LEITE et al., 1997).

As pesquisas realizadas por Muller et al. (2005), Reiner et al. (2012), Leles et al. (2001), Silva (2005), Sereghetti et al. (2015) dentre outros estudos, reafirmaram que a maior produtividade volumétrica ocorre nos plantios mais adensados, ou seja, nos casos de maior densidade populacional por hectare.

De maneira geral, os resultados deste estudo apontam para o planejamento da produção em função do espaçamento de plantio. Quando se deseja obter árvores de maior diâmetro, maior volume individual, o indicado é a adoção de espaçamentos mais amplos e com rotações mais longas. Por outro lado, os espaçamentos menores entre plantas devem ser priorizados para os sistema de curta rotação para fins energéticos. Esse conceito visa alta produção volumétrica por unidade de área, em menor espaço de tempo, para produção de biomassa (GUERRA et al., 2012). Além disso, conforme sugerem Lopes et al. (2017), a partir dos resultados de estudo, ao avaliar diferentes espaçamentos e clones de Corymbia e Eucalyptus para energia, sobre a necessidade de considerar as características dendrométricas, químicas e de massa especifica dos diferentes materiais genéticos de forma integrada, quando se busca a produção de bioenergia.

Em situações que o silvicultor não tem um destino final definido para a produção florestal, o mais adequado é que sua escolha esteja entre espaçamentos com lotações intermediárias (1666 a 2500 plantas ha ${ }^{-1}$ ). Neste caso, os custos de implantação e condução da floresta e as alternativas do regime de manejo mais indicadas também devem ser previstas para que se garanta um bom retorno do empreendimento.

\section{Conclusões}

Houve efeito do espaçamento de plantio sobre as variáveis dendrométricas e a produção florestal na idade avaliada.

O diâmetro, a área basal e o volume mostraram-se correlacionadas e dependentes do espaçamento de plantio.

Maiores valores médios de altura, diâmetro, área transversal e volume individual ${ }^{-1}$ foram verificados nos espaçamentos mais amplos.

Os espaçamentos mais adensados favoreceram os valores médios de área basal ha ${ }^{-1}$ e a produção $h a^{-1}$.

\section{Agradecimentos}

À Empresa Golden Tree Reflorestadora Ltda., pela cessão das mudas utilizadas na implantação do plantio experimental estudado nesta pesquisa.

\section{Referências}

ALZATE, S.B.A.; TOMAZELLO FILHO, M.; PIEDADE, S.M. de S. Variação longitudinal da densidade básica da madeira de clones de Eucalyptus grandis Hill ex Maiden, E. saligna Sm. e E. grandis $x$ urophylla. Scientia Forestalis, Piracicaba, v.68, p.87-95, 2005.

BERGER, R.; SCHNEIDER, P. R.; FINGER, C. A. G.; HASELEIN, C.R. Efeito do espaçamento e da adubação no crescimento de um Clone de Eucalyptus saligna Smith. Ciência Florestal, Santa Maria, v.12, n.2, p.75-87, 2002. http://dx.doi.org/10.5902/198050981682.

BINKLEY, D.; STAPE, J.L; RYAN, M. G.; BARNARD, $\mathrm{H}$. Age- related decline in forest ecosystem growth: an individual- tree, stand- structure hypothesis. Ecosystems, New York, v.5, p.58-67, 2002. https://doi.org/10.1007/s10021-001-00557

EMBRAPA FLORESTAS. Mapa simplificado de solos do estado do paraná. Disponível em: http://www.escola.agrarias.ufpr.br/arquivospdf/ mapa_solos_pr.pdf. Acesso em: 24 set. 2018.

ENCINAS, J. I.; SILVA, G. F.; PINTO, J. R.R. Idade e crescimento das árvores. Comunicações Técnicas Florestais, Brasília: UFB, 2005. v.7, n.1, 25p.

GRAÇA, M.E.C. et al. Capacidade de rebrota e de enraizamento de Eucalyptus benthamii. Boletim de Pesquisa Florestal, n.39, p.135-138, 1999.

GUERRA, S. P. S.; LANÇAS, K. P.; GARCIA, É. A.; SPINELLI, R. Bioenergia, desenvolvimento, pesquisa e inovação. In: LEMOS, E. G. M.; STRADIOTTO, N. R. Bioenergia: desenvolvimento, pesquisa e inovação. São Paulo: Unesp, 2012. Cap. 6. p. 126-162. 
HIGA, R. C. V.; PEREIRA, J. C. D. Usos potenciais do Eucalyptus benthamii Maiden et Cambage. Colombo: Embrapa Florestas, 2003. (Comunicado Técnico; $\left.n^{\circ} 100\right)$.

IBA. Relatório anual 2020. Disponível em: https://iba.org/datafiles/publicacoes/relatorios/i ba-relatorioanual2020. Acesso em: 25 out. 2020.

INOUE, M. T.; FIGUEIREDO FILHO, A.; LIMA, R. Influência do espaçamento de crescimento na altura e diâmetro de Pinus taeda L. Scientia Forestalis, Piracicaba, v.39, n.91, p.377-385, 2011.

KRONKA, F. J. N.; BERTOLANI, F. PONCE, R.H. A cultura do pinus no Brasil. São Paulo: Sociedade Brasileira de Silvicultura, 2005.

LELES, P. S. S.; REIS, G. G. FEREIRA REIS, M. G.; MORAIS, E. J. Crescimento, produção e alocação de matéria seca de Eucalyptus camaldulenis e $E$. pellita sob diferentes espaçamentos na região de Cerrado, MG. Scientia Forestalis, Piracicaba, n.59, p.77-87, 2001.

LEITE, F. P.; BARROS, N. F.; NOVAIS, R. F.; SANS, L. M. A.; FABRES, A. S. Crescimento de Eucalyptus grandis em diferentes densidades populacionais. Revista Árvore, Viçosa, v.21, n.3, p.313-321, 1997.

LEITE, H. G.; NOGUEIRA, G.S.; MOREIRA, A. M. Efeito do espaçamento e da idade sobre variáveis de povoamentos de Pinus taeda L. Revista Árvore, Viçosa, v.4, n.30, p.603-612, 2006. http://dx.doi.org/10.1590/S0100$\underline{67622006000400013}$

LEITE, E. S.; MINETTE, L. J.; FERNANDES, H. C.; SOUZA, A. P.; AMARAL, E. J.; LACERDA, E. G. Desempenho do harvester na colheita de eucalipto em diferentes espaçamentos e declividades. Revista Árvore, Viçosa, v.38, n.1, p.000-000, 2014. https://doi.org/10.1590/S0100$\underline{67622014000100009}$.

LIMA, R.; INOUE, M. T.; FIGUEIREDO FILHO, A.; ARAUJO, A. J.; MACHADO, S. A. Efeito do espaçamento no desenvolvimento volumétrico de Pinus taeda L. Floresta e Ambiente, Seropédica, v.20, n.2, p.223-230, 2013. https://doi.org/10.4322/floram.2013.001
LOPES, E. D.; LAIA, M. L.; SANTOS, A. S.; SOARES, G. M.; LEITE, R. W. P.; MARTINS, N. S. Influência do espaçamento de plantio na produção energética de clones de Corymbia e Eucalyptus. Floresta, Curitiba, v.47, n.1, p.95-104, jan./mar. 2017. DOI: $10.5380 /$ rf.v47i1.47141

MAGALHÃES, W. S.; MACEDO, R. L. G.; VENTURIM, N.; HIGASHIKAWA, E. M.; YOSHITANI JUNIOR, M. Desempenho silvicultural de espécies de Eucalyptus spp. em quatro espaçamentos de plantio na região noroeste de Minas Gerais. Floresta e Ambiente, Seropédica, v.12, n.2, p.0107, 2006.

MULLER, M. D.; COUTO, L.; LEITE, H. G.; BRITO, J. O. Avaliação de um clone de Eucalypto estabelecido em diferentes densidades de plantio para a produção de biomassa e energia. Biomassa e Energia, Viçosa, v.2, n.3, p. 177-186, 2005.

MORAIS, V. Dinâmica de crescimento de eucalipto sob diferentes espaçamentos, na região noroeste do estado de Minas Gerais. 2006. Dissertação (Mestrado) - Universidade Federal de Lavras, Lavras-MG.

PINKARD, E. A.; NEILSEN, W. A. Crown and stand characteristics of Eucalyptus nitens in response to initial spacing: implications for thinning. Forest Ecology and Management, v.172, n.2/3, p.215227, 2003. https://doi.org/10.1016/S03781127(01)00803-9

PEREIRA, J. C. D.; STURION, J. A.; HIGA, A. R.; HIGA, R. C. V.; SHIMIZU, J. Y. Características da madeira de algumas espécies de eucalipto plantadas no Brasil. Colombo: Embrapa Florestas, 2000. 113 p. (Documentos; 38).

REINER, D. A.; SILVEIRA, E. R.; SZABO, M. S. O uso de Eucalyptus em diferentes espaçamentos como alternativa de renda e suprimento da pequena propriedade na região Sudoeste do Paraná. Synergismus Scyentifica, Pato Branco, v.6, n.1, $\mathrm{s} / \mathrm{n}, 2011$.

REINER, D. A.; SILVEIRA, E. R.; FERRONATO, M. L.; MENEGASSI, A. D. Desenvolvimento de Eucalyptus benthamii em diferentes espaçamentos de plantio em Pato BrancoParaná. In: Congresso Florestal Paranaense, 4. Anais [...]. Curitiba, 2012. 
SANTOS, M. D. Efeito do espaçamento de plantio na biomassa do fuste de um clone hibrido interespecífico de Eucalyptus grandis e Eucalyptus urophylla. 2011. 140p. Dissertação (Mestrado). Faculdade de Ciências Agronômicas da UNESP, Botucatu-SP.

SILVA, C. R. Efeito do espaçamento e arranjo de plantio na produtividade e uniformidade de clones de Eucalyptus na região nordeste do Estado de São Paulo. 2005. 51p. Dissertação (Mestrado em Ciência Florestal) - Escola Superior de Agricultura "Luiz de Queiroz", Universidade de São Paulo, Piracicaba-SP.

SANQUETA, C. R.; MORA, A. L.; BORSATO, R.; VIDAL, M. A. S.; PEIXOTO, A, M.; CHIARANDA, R. Efeito do espaçamento de plantio em reflorestamentos - II. Pinus taeda L. em Jaguariaíva - PR. Revista Acadêmica: Ciências Agrárias e Ambientais, Curitiba, v.1, n.1, p. 5561, 2003. https://doi.org/10.7213/cienciaanimal.v1i1.1488 $\underline{9}$

SCHNEIDER, P. R. Introdução ao manejo florestal. Santa Maria: UFSM, CEPEF, FATEC. 1993. 348p.

SEREGHETTI, G. C.; LANÇAS, K. P.; SARTORI, M. S.; REZENDE, M. A.; SOLER, R. R. Efeito do espaçamento no crescimento e na densidade básica da madeira de eucalyptus urophylla $\mathrm{x}$ Eucalyptus grandis em florestas de ciclo curto. Energia Agricultura, Botucatu, v.30, n.3, p.257262, 2015. https://doi.org/10.17224/EnergAgric.2015v30n3p $\underline{257-262}$

SCHUMACHER, F.; HALL, F. Logarithmic expression of timber- tree volume. Journal of agricultural research, v.47, p.719 -734, 1933. 